九州大学学術情報リポジトリ

Kyushu University Institutional Repository

\title{
Machine-Learning-Based 0 lfactometer: Prediction of Odor Perception from Physicochemical Features of Odorant Molecules
}

\section{Shang, Liang}

Department of Electronics, Graduate School of Information Science and Electrical Engineering, Kyushu University

Liu, Chuanjun

Department of Electronics, Graduate School of Information Science and Electrical Engineering, Kyushu University | Research Laboratory, U.S.E. Co., Ltd.

Tomiura, Yoichi

Department of Informatics, Graduate School of Information Science and Electrical Engineering, Kyushu University

Hayashi, Kenshi

Department of Electronics, Graduate School of Information Science and Electrical Engineering, Kyushu University

http://hdl. handle. net/2324/1932613

出版情報: Analytical Chemistry. 89 (22)，pp.11999-12005，2017-10-13. American Chemical Society バージョン：

権利関係 : 


\title{
Machine-learning-based Olfactometer: Prediction of Odor
}

\section{Perception from Physicochemical Features of Odorant Molecules}

\author{
Liang Shang, ${ }^{\dagger}$ Chuanjun Liu, ${ }^{\dagger, \S}$ Yoichi Tomiura, ${ }^{\ddagger}$ Kenshi Hayashi ${ }^{*}{ }^{\dagger}$ \\ 'Department of Electronics, Graduate School of Information Science and Electrical Engineering, Kyushu University, \\ Fukuoka 819-0395, Japan \\ ${ }^{\S}$ Research Laboratory, U.S.E. Co., Ltd., Tokyo 150-0013, Japan \\ *Department of Informatics, Graduate School of Information Science and Electrical Engineering, Kyushu University, \\ Fukuoka 819-0395, Japan
}

\begin{abstract}
Gas chromatography-olfactometry (GC-O) has been used in various fields as a valuable method to identify odor-active components from a complex mixture. Since human assessors are employed as a detector to obtain the olfactory perception of separated odorants, the GC-O technique is limited by its subjectivity, variability, and high cost of the trained panelist. In this paper, we present a proof-of-concept model by which odor information can be obtained not from the human panelist but rather the physicochemical features of odorant molecules. Models were established using a database of flavors and fragrances including 1026 odorants and corresponding verbal odor descriptors (ODs). Physicochemical parameters of the odorant molecules were acquired using the "Dragon" software. Ten representative ODs were selected to build the prediction models based on their high occurrence frequency in the database. The features of the molecular parameters (MPs) were extracted using either unsupervised (principle component analysis) or supervised (Bortua: BR) approaches, and then used as input to calibrate machine learning models. The synthetic minority oversampling technique was used to compensate for any imbalance problems of the analyzed database. Predictions were performed using various machine learning approaches such as support vector machine (SVM), random forest, and extreme learning machine. All models were optimized via parameter tuning and their prediction accuracies were compared. A SVM model combined with feature extraction by BR-C (confirmed only) was found to afford the best results with a calibration accuracy of $97.08 \%$ and a prediction accuracy of $97.08 \%$. This result demonstrated that the odor prediction model might be used to replace human panelists to fabricate the machine-learning-based GC-O.
\end{abstract}

Gas chromatography-olfactometry (GC-O) has been developed as a powerful tool in the field of odor research because of the coupled performance of gas chromatographic analysis with human panelist sensory detection ${ }^{1,2}$. GC-O can work not only as an instrumental analysis to identify and quantify complex odor mixtures, but also as a sensorial analysis to assess aroma or odor-active compounds within the GC effluents ${ }^{3}$. In GC-O analysis, the eluted substances are perceived simultaneously by two detection systems; one is a mass spectrometry (MS) system and the other is the human olfactory $\operatorname{system}^{4-6}$. Evaluation by a human sniffer plays an important role because it can make up for deficiencies of GC (or GC-MS) in odor analysis ${ }^{7}$. For example, many of peaks detected by GC for an odor mixture may not actually contribute to our perception since they are present below our thresholds for detecting them. Conversely, some compounds may not show up as detectable GC peaks, but may have a low perception threshold and contribute substantially to a sample's profile. The sensory evaluation of smells by trained panelists can overcome such problems and represents a valid approach to odor assessment. Through sniffing GC effluent components, panelists can determine the odor characteristics ascribed to each individual component, which is important information for the overall odor analysis ${ }^{8}$. 
A major problem of GC-O is the subjectivity of assessors at the intra- and inter- individual level. Sensory assessment of smells by panelists is influenced by many factors, such as the testing environment, experimental bias, assessor sensitivity, assessor selection, and training ${ }^{9}$. Experimental conditions should be well established to ensure accuracy and precision of the odor descriptor data collected by the panelist. Therefore, although GC-O has presented many challenges not considered on typical GC analysis, its application and promotion are hindered by the variability, high technical requirements, and high costs of the trained panelist ${ }^{10-12}$. Some software modules have been designed and applied in GC-O as a supplement to aroma and chemical analysis. For example, AroChemBase (Alpha MOS, Japan) consists of the most comprehensive chemical and sensory library ever, which is convenient for fast sensory profiling and detailed chemical/aroma characterization. In this kind of software module, however, the number of compounds with odor descriptors (around 2000) is far less than that of the total compound pool (around 44000). Therefore, regardless of human assessment or software indexing, the question of how to effectively obtain sensory information for eluted compounds from $\mathrm{GC}$ is still unanswered for GC-O.

Recently, much effort has been focused on clarifying the structure-odor relationship using structural, topological, geometrical, electronic, and physicochemical parameters of odorant molecules ${ }^{13}$. The driving force of this research might come from the great progress made by Buck and Axel through their discoveries of odorant receptors and the organization of the olfactory system ${ }^{14,15}$. Many studies have revealed that odorants with similar molecular features show similar activity patterns in the olfactory bulb (also referred to as the odor map $)^{16}$. Althoug it is still difficult to establish a general rule to predict the olfactory perception of odorants, a number of computational techniques have been used successfully in the explanation of both physicochemical and perceptual spaces of odorant molecules. For example, Sobel et al. related these two spaces to each other and find that the primary axis of perception (defined as odor pleasantness), reflects the primary axis of physicochemical features. Kumar et al. developed a network based approach (smell network) which can be used to explore the perceptual universe and prove the underlying similarity of percepts ${ }^{17,18}$. Keller et al. established a machine-learning algorithm using a large olfactory psychophysical data set, which can be used to predict odor intensity, pleasantness, and semantic descriptors from chemical features of odor molecules ${ }^{19}$.

The above recent developments demonstrate that it becomes realistic to predict olfactory perception from structural parameters of odorant molecules. The development of data analysis techniques and cheminformatics software make it possible to deal with the complexity of odor spaces that have high dimensionality and nonlinearity. However, to our knowledge no study has applied the odor prediction models in GC-O. In this proof-of-concept study, we test the possibility that a machine-learning based prediction model could be used to replace the human panelist in GC-O. As illustrated in Figure 1, after the GC effluent is identified by mass spectrometry, its molecular parameters (MP) can be transferred by a cheminformatics software and inputted into a classifier system in which each classifier is labeled by a specific odor descriptor (OD) (a word like "sweet", "green", "fruity", and "herbaceous", etc.). After the true or false classification, the system can output the sensory information of the GC effluent, which may consist of single OD (such as sweet) or multiple ODs (such as sweet and green).

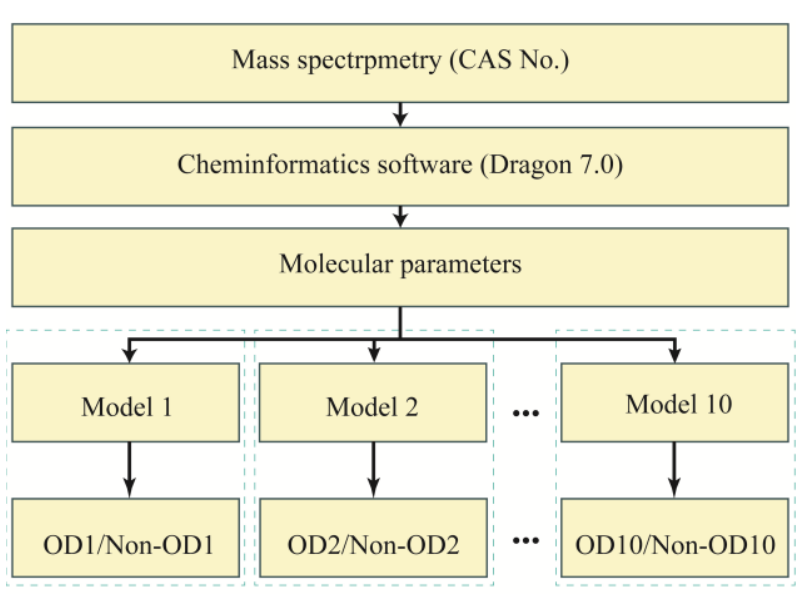

Figure 1. Concept diagram to predict odor descriptors using molecular parameters.

A flavor and fragrance database (Sigma-Aldrich, 2016) that includes 1026 odorants and 10 ODs was considered in this study. The physicochemical MPs were acquired via a cheminformatics software. The features of the MPs were extracted using either unsupervised (principle component analysis: PCA) or supervised (Bortua: BR) approaches. Ten typical ODs with high occurrence frequency in the database were selected to establish the models. Differernt machine 
learning algorithms, including support vector machine (SVM), random forest $(\mathrm{RF})$, and extreme learning machine (ELM), were used and their prediction results were compared. A Bortua-SVM model showed high accuracy in ODs prediction, which indicates the possibility for machine-learning based GC-O.

\section{- MATERIALS AND METHODS}

Odor Data Collection. Simplified molecular input line entry specification (SMILES) were obtained by both semi-automatic and manual methods from PubChem (https://pubchem.ncbi.nlm.nih.gov/) according to the CAS number of the odorant molecules recorded in the Flavors \& Fragrances database (Sigma-Aldrich, 2016). The SMILES strings were imported into the Dragon chemoinformation software (version 7.0, Kode, Italy) to compute the physicochemical parameters. The calculation afforded 5270 parameters with various values for each odorant molecule. It was found that most of parameters (around 4200) were assigned with not applicable (NA). We removed these parameters with "NA", and finally got a parameter matrix with 1006 MPs. All MPs were normalized and centered for further processing.

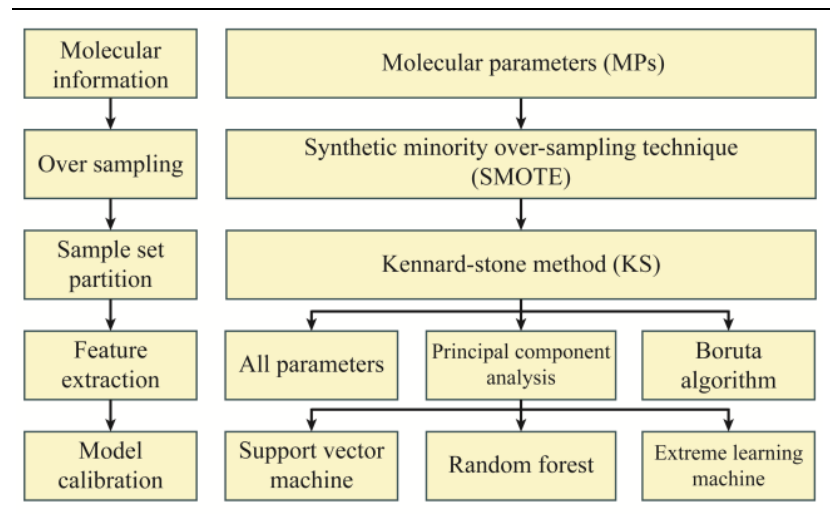

Figure 2. Data processing diagram of prediction models calibration.

Data Analysis. The data analysis process is shown in Figure 2. The dataset for odor prediction is a typical imbalanced dataset because the class distribution of the positive samples (minor samples with specified ODs labels) and the negative samples (major samples with non-specified ODs labels) is not uniform. Here, synthetic minority oversampling technique (SMOTE) was employed to overcome the imbalance problem $^{20}$. The minority class was over-sampled at $300 \%$ of its original size and the majority class was under-sampled to obtain a balanced dataset.
Afterwards, the sample pool was divided into training and test sets with a 3:1 ratio using the Kennard-Stone (KS) algorithm $^{21,22}$. Sample size details for each OD are listed in Table 1. The unsupervised feature combination method (PCA) and supervised feature selection method (Boruta) were performed to extract kernel information to enhance the performance of the classification frameworks ${ }^{23,24}$. SVM, RF, and ELM classification algorithms were applied to predict ODs. The optimal model was determined by considering the accuracies of the training and test sets. As the last step, the F1 score based on precision and recall was used to verify the performance of the optimal model ${ }^{25}$.

\section{RESULTS AND DISCUSSION}

Odor Descriptors. It is well known that for machine learning, the larger the sample size is, the higher the model accuracy tends to be. For odor prediction, an optimum database should have an appropriate number of odorant molecules and ODs. Up to now, there are a number of odor databases that have been reported and analyzed ${ }^{26}$. Recently, Kumar et. al., carried out a comprehensive statistical analysis of 5 main odor databases which include Flavornet, goodScents, Leon \& Johnson, Sigma-Aldrich, and SuperScent ${ }^{18}$. One problem of these databases is the sparseness of the data distribution because an odorant molecule can be described by a varying number of ODs, but very few molecules are described by a large number of ODs in the databases. The statistical results of Kumar indicate that the Sigma-Aldrich database possesses both a relatively larger number of odorant molecules and larger average number of ODs per molecule, and thus leads to the highest average occurrence of ODs. In view of this characteristic, the Flavors \& Fragrances database of Sigma-Aldrich (2016), which has been upgraded to 1026 odorant molecules and 160 ODs, was adopted and analyzed in the present study. Detailed information about the 160 ODs is listed in Table S1. Figure 3 summarizes the 20 ODs that occurred most frequently in the database. The descriptor of "Sweet" is represented by approximately 200 odorants while the descriptor of "Rose" is represented by approximately 50 odorants. Considering that a badly-calibrated model could result from insufficient sampling, only the top 10 ODs were used to establish our prediction models. The 10 descriptors include sweet, green, fruity, floral, meaty, wine-like, apply, fatty, woody, and herbaceous. The minimum number of 
samples (herbaceous) is over 70. This sample size may help ensure accuracy of the prediction models.

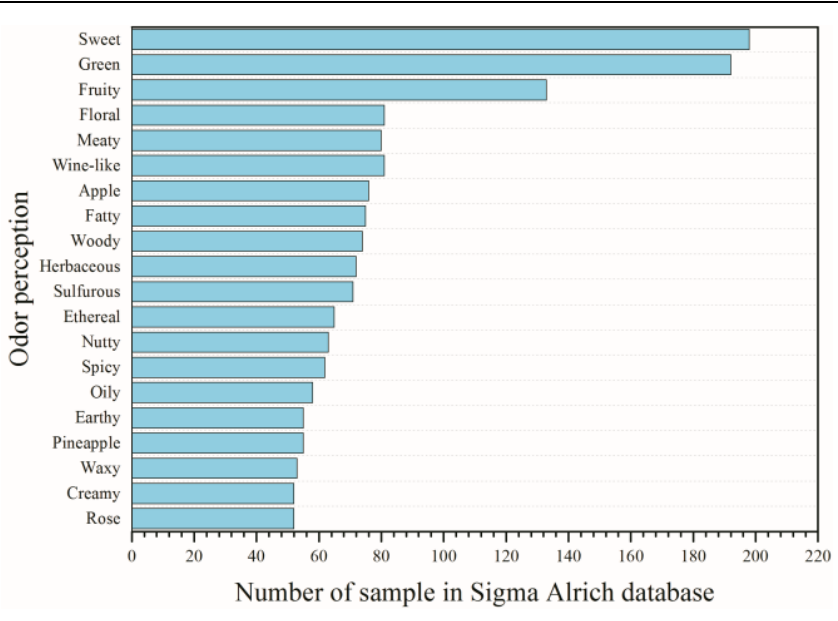

Figure 3. The most 20 frequent ODs in Sigma-Aldrich database. Considered the sample size for model calibration, the firstly ten odor descriptors were considered in this study.

Feature extraction. As machine learning aims to deal with larger, more complex questions, the extraction of relevant features for data representation data is a critical problem within model calibration ${ }^{27}$. It has been reported that machine learning algorithms exhibit a decrease of accuracy when the number of variables is significantly higher than an optimal number $^{28}$. Consequently, before model calibration, PCA and BR were employed using unsupervised and supervised methods, separately, to extract features from all the MPs, and their effects were evaluated.

PCA was first performed to remove redundant information (Figure S1). To avoid loss characteristic information from the original data set, PCs with accumulative contributions of 99.99\% were selected. Table 1 lists the number of PCs for 10 ODs. BR was used to find useful features of each OD. By BR, 1006 MPs were labeled as 'confirmed', 'tentative', or 'rejected' (Table 1). In this research, MPs labeled 'confirmed' or 'tentative' (BR-CT), and labeled 'confirmed' only (BR-C) were used in further processing. Features selected by BR for the 10 ODs are shown in Figure 4. This illustrates that although an MP may be labeled as 'confirmed' for one OD, the MP could be regarded as a useless feature for other ODs. This indicates that ODs could be used to describe various dimensions for an odorant. It can be interpreted that some MPs are associated with some appointed functional groups of a molecule, and functional groups are related to ODs.
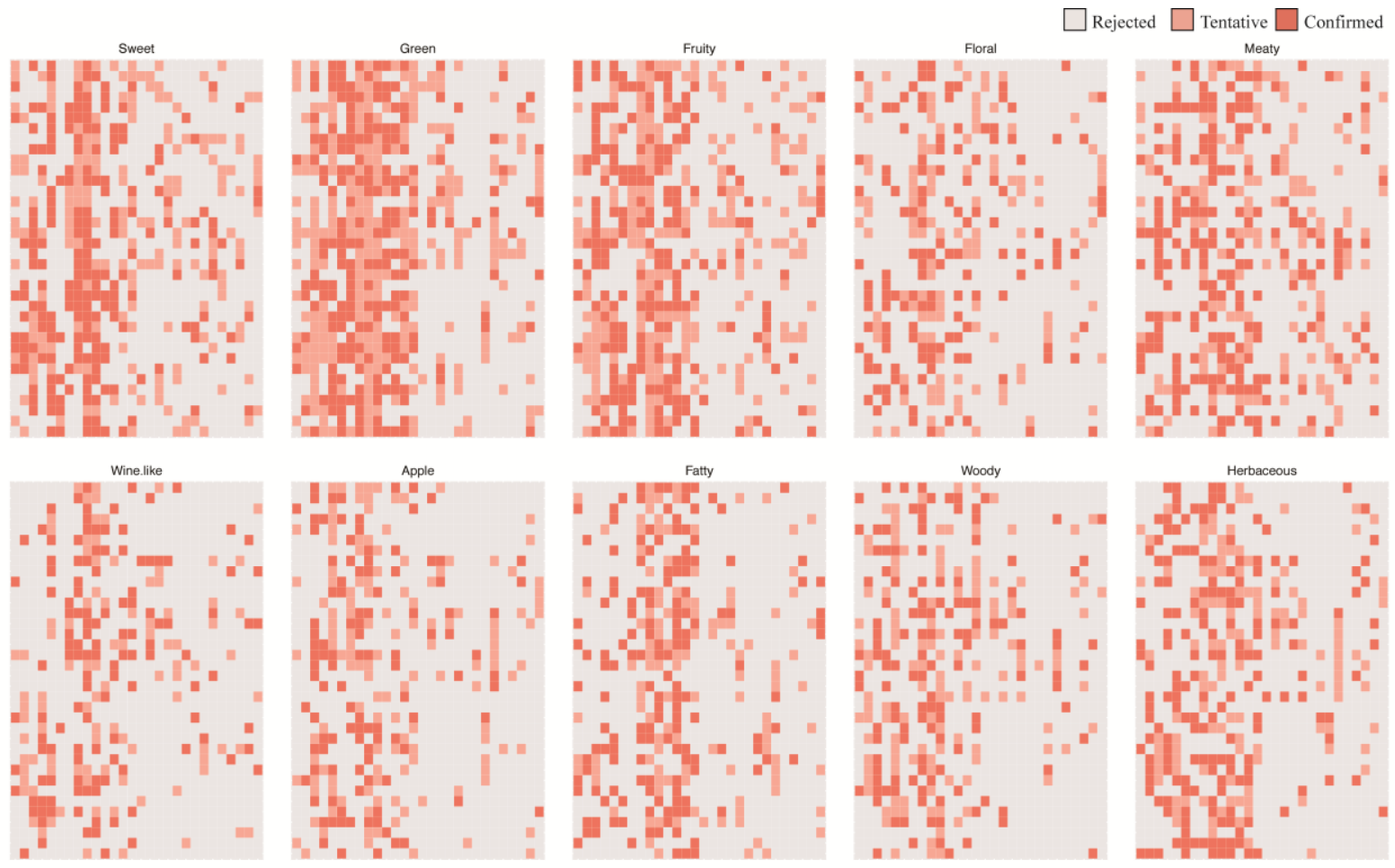

Figure 4. MPs selection based on BR method for the ten ODs. 1006 MPs were arranged as a matrix $(36 \times 28)$. Each grid indicated one MP. MPs labeled as 'rejected', 'tentative' or 'confirmed' were colored. Here, the features labeled 'tentative' and 'confirmed' (BR-CT) and only labeled 'confirmed' (BR-C) were used for calibrating models. The number of MPs for each label were listed in Table 1. 
Table 1. Data descriptions for original dataset, SMOTE processed dataset, division of samples by KS, the number PCs and MPs labeled by BR method.

\begin{tabular}{|c|c|c|c|c|c|c|c|c|c|c|c|}
\hline \multirow{2}{*}{$\begin{array}{c}\text { Odor } \\
\text { descriptor }\end{array}$} & \multicolumn{3}{|c|}{ Original dataset } & \multicolumn{2}{|c|}{ SMOTE processed dataset } & \multicolumn{2}{|c|}{ Division of samples by KS } & \multirow{2}{*}{ Number of PCs } & \multicolumn{3}{|c|}{ MPs labeled by BR method } \\
\hline & P sample ${ }^{*}$ & N sample & $\mathrm{F}: \mathrm{T}$ & P sample & T sample & Train set & Test set & & Confirmed & Tentative & Rejected \\
\hline Sweet & 198 & 828 & $4.18: 1$ & 792 & 891 & 1262 & 421 & 260 & 180 & 206 & 620 \\
\hline Green & 192 & 834 & $4.34: 1$ & 768 & 864 & 1224 & 408 & 267 & 263 & 211 & 532 \\
\hline Fruity & 133 & 893 & $6.71: 1$ & 532 & 598 & 847 & 283 & 238 & 214 & 205 & 587 \\
\hline Floral & 81 & 945 & $11.67: 1$ & 324 & 364 & 516 & 172 & 201 & 118 & 136 & 752 \\
\hline Meaty & 80 & 946 & 11.83:1 & 320 & 360 & 510 & 170 & 201 & 141 & 208 & 657 \\
\hline Wine-like & 81 & 945 & $11.67: 1$ & 324 & 364 & 516 & 172 & 200 & 95 & 126 & 785 \\
\hline Apple & 76 & 950 & $12.50: 1$ & 304 & 342 & 484 & 162 & 188 & 133 & 109 & 764 \\
\hline Fatty & 75 & 951 & $12.68: 1$ & 300 & 337 & 477 & 160 & 199 & 116 & 155 & 735 \\
\hline Woody & 74 & 952 & $12.86: 1$ & 296 & 333 & 471 & 158 & 189 & 129 & 132 & 745 \\
\hline Herbaceous & 72 & 954 & $13.25: 1$ & 288 & 324 & 459 & 153 & 188 & 122 & 177 & 707 \\
\hline
\end{tabular}

*P (Positive) sample indicates the number of samples with the specific OD label. N (Negative) sample indicates the number of samples with the non-specific OD label.

SVM Model. The 5-fold cross validation was applied to select the penalizing factor $(c)$ and RBF kernel parameter $(g)$ to develop SVM models in this research. By multiple attempts, the range of $c$ was set from $2^{0}$ to $2^{-20}$ (the range of $g$ was set from $2^{-10}$ to $2^{10}$ ) with an increment of $2^{0.5}$. The optimal values for SVMs were confirmed by the highest accuracy in all combination of $c$ and $g$ (Figure S2). The selected results for the ten ODs are listed in Table 2. The identification accuracies for the calibration set and the validation set of AP-SVM, PCA-SVM, BR-CT-SVM, and BR-C-SVM are shown in Table S2. The results indicated that all the models calibrated by SVM had a similar average accuracy: $96.83 \pm 1.7 \%$ for AP-SVM, $96.89 \pm 1.9 \%$ for PCA-SVM, 97.19 $\pm 0.93 \%$ for BR-CT-SVM, and $96.10 \pm 2.8 \%$ for BR-C-SVM. However, considering the complexity of the model, BR-C-SVM should be regarded as the optimal model for the present study.
RF Model. To calibrate RF models, two parameters: the number of trees $\left(n_{\text {tree }}\right)$ and the number of features $\left(m_{t r y}\right)$ need to be optimized. Although adding more trees will not cause over-fitting, it will increase the model complexity. Therefore, a sufficient number of trees was needed to calibrate RF models. Considered the out of bag error and test error, the optimal $n_{\text {tree }}$ and $m_{\text {try }}$ were determined (Figure S3, S4). The optimal modeling parameters of the RF models are listed in Table 2. The overall accuracies of the best RF models under AP, PCA, BR-CT, and BR-C datasets are shown in Figure 4. In summary, PCA-RF showed a better average accuracy $(92.79 \pm 1.63 \%)$ than the AP-RF $(90.62 \pm 1.26 \%)$, BR-CT-RF $(90.50 \pm 1.21 \%)$, and BR-C-RF $(90.61 \pm 1.85 \%)$ models.

\section{Table 2. Modeling parameters for SVM, RF and ELM models calibration.}

\begin{tabular}{|c|c|c|c|c|c|c|c|c|c|c|c|c|c|c|c|c|c|c|c|c|}
\hline \multirow{2}{*}{$\begin{array}{c}\text { Odor } \\
\text { descriptor }\end{array}$} & \multicolumn{5}{|c|}{$\mathrm{AP}$} & \multicolumn{5}{|c|}{ PCA } & \multicolumn{5}{|c|}{ BR-CT } & \multicolumn{5}{|c|}{ BR-C } \\
\hline & $c^{*}$ & $g$ & $\mathrm{~m}_{\text {try }}$ & $\mathrm{n}_{\text {tree }}$ & $\mathrm{n}_{\text {hidden }}$ & $c$ & $g$ & $\mathrm{~m}_{\text {try }}$ & $\mathrm{n}_{\text {tree }}$ & $\mathrm{n}_{\text {hidden }}$ & $c$ & $g$ & $\mathrm{~m}_{\text {try }}$ & $\mathrm{n}_{\text {tree }}$ & $\mathrm{n}_{\text {hidden }}$ & $c$ & $g$ & $\mathrm{~m}_{\text {try }}$ & $\mathrm{n}_{\text {tree }}$ & $\mathrm{n}_{\text {hidden }}$ \\
\hline Sweet & 1.414 & 0.006 & 46 & 187 & 660 & 1.414 & 0.008 & 160 & 126 & 794 & 1.414 & 0.011 & 96 & 39 & 575 & 1.414 & 0.022 & 90 & 52 & 387 \\
\hline Green & 2.828 & 0.004 & 186 & 34 & 575 & 2.000 & 0.008 & 27 & 97 & 796 & 2.000 & 0.008 & 124 & 56 & 633 & 5.657 & 0.022 & 103 & 32 & 440 \\
\hline Fruity & 2.828 & 0.008 & 86 & 90 & 537 & 4.000 & 0.008 & 128 & 91 & 626 & 1.414 & 0.011 & 99 & 32 & 508 & 256 & 0.022 & 54 & 25 & 399 \\
\hline Floral & 1.414 & 0.006 & 36 & 93 & 320 & 1.414 & 0.006 & 21 & 133 & 792 & 1.000 & 0.022 & 34 & 53 & 230 & 1.414 & 0.011 & 28 & 218 & 204 \\
\hline Meaty & 1.000 & 0.001 & 346 & 75 & 326 & 1.000 & 0.001 & 21 & 59 & 795 & 2.000 & 0.006 & 109 & 31 & 321 & 2.828 & 0.004 & 91 & 24 & 271 \\
\hline Wine-like & 4.000 & 0.006 & 26 & 32 & 347 & 4.000 & 0.006 & 130 & 170 & 786 & 2.000 & 0.022 & 11 & 36 & 309 & 1.414 & 0.088 & 75 & 66 & 322 \\
\hline Apple & 1.414 & 0.011 & 196 & 54 & 312 & 1.414 & 0.011 & 128 & 37 & 795 & 1.414 & 0.022 & 172 & 57 & 244 & 1.414 & 0.088 & 123 & 36 & 335 \\
\hline Fatty & 8.000 & 0.002 & 666 & 27 & 255 & 8.000 & 0.002 & 129 & 106 & 786 & 4.000 & 0.006 & 51 & 42 & 220 & 1.414 & 0.031 & 96 & 34 & 180 \\
\hline Woody & 4.000 & 0.008 & 46 & 92 & 295 & 2.828 & 0.008 & 69 & 107 & 797 & 1.414 & 0.022 & 121 & 61 & 270 & 1.000 & 0.044 & 79 & 64 & 217 \\
\hline Herbaceous & 4.000 & 0.008 & 36 & 56 & 328 & 8.000 & 0.011 & 78 & 87 & 768 & 2.000 & 0.044 & 29 & 74 & 305 & 2.828 & 0.063 & 82 & 20 & 724 \\
\hline
\end{tabular}

${ }^{*}$ The penalizing factor $(c)$ and RBF kernel parameter $(g)$ for SVM models, number of features ( $\left.m_{\text {try }}\right)$ and number of trees ( $\left.n_{\text {tree }}\right)$ for RF models and number of hidden nodes ( $\mathrm{n}_{\text {hidden }}$ ) for ELM models. 
ELM Model. For ELM models, the parameter need to be tuned is the number of hidden layer nodes. In this study, the parameter was obtained by a trial and error method. The range of number of hidden nodes was set from 1 to 800 . To avoid the randomness of ELM models, each ELM model was repeated 200 times and the average accuracy was employed to finish the parameter's selection (Figure S5). Based on the highest average accuracies of calibration set and validation set, the optimal parameter was determined. Selected results are provided in Table 2. The ODs identification accuracies for the training and test sets of ELM are shown in Figure 4. It shows that the accuracy of PCA-ELM $(97.53 \pm 1.35 \%)$ is higher than AP-ELM (94.13 $\pm 1.44 \%)$, BR-CT-ELM $(94.02 \pm 1.59 \%)$, and BR-C-ELM $(91.78 \pm 1.91 \%)$.

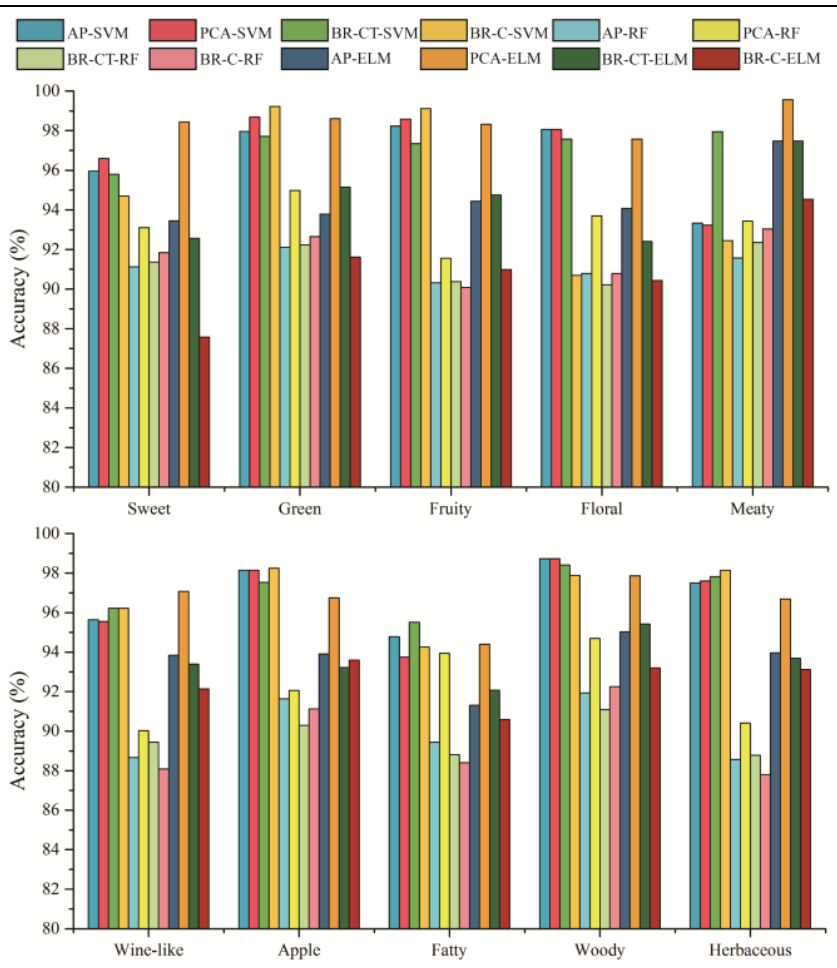

Figure 5. Identification average accuracies of train and test sets for ten odor descriptors by SVM, RF and ELM.

Model Comparison. The average accuracies of the training and test sets for ODs are shown in Figure 5. For 'green', 'fruity', 'wine-like', 'apple', and 'herbaceous' identification, BR-C-SVM shows better results than other models. However, PCA-ELM did a better job identifying the 'sweet' and 'meaty' ODs. When the tree modeling methods were compared (Figure 6 ), it was found that the ELM had the best identification accuracy (97.53 $\pm 1.35 \%)$, followed by SVM $(97.19 \pm 0.93 \%)$, and RF $(92.79 \pm 1.63 \%)$. Dealing with large variables slows down machine learning algorithms and requires more resources $^{29}$. Here, PCA and BR were employed to extract kernel information from a large feature set. The results show that PCA did a better job than Boruta in the RF and ELM models. However, PCA is an unsupervised feature combination method; the PCs are computed based on original data set. Considering the amount of input information, BR is more suitable for feature extraction from MPs. It was confirmed that the training time increases with the number of features. Here, by the BR-C method, only $15.01 \%$ information was extracted instead of all MPs. Therefore, considering the accuracies and modeling time comprehensively, it is suggested that SVM combined with features extracted by BR-C, whose average accuracy was higher than $96.10 \pm 2.8 \%$, is the optimal model in identifying perceptual descriptors based on MPs. Besides, the recall, precision, and F1 score of BR-C-SVM were $94.83 \pm 5.61 \%, 86.88 \pm 3.04 \%$, and $95.74 \pm 3.52 \%$, respectively, which yields the model with an acceptable generalization ability to predict odor descriptors based on physicochemical parameters.

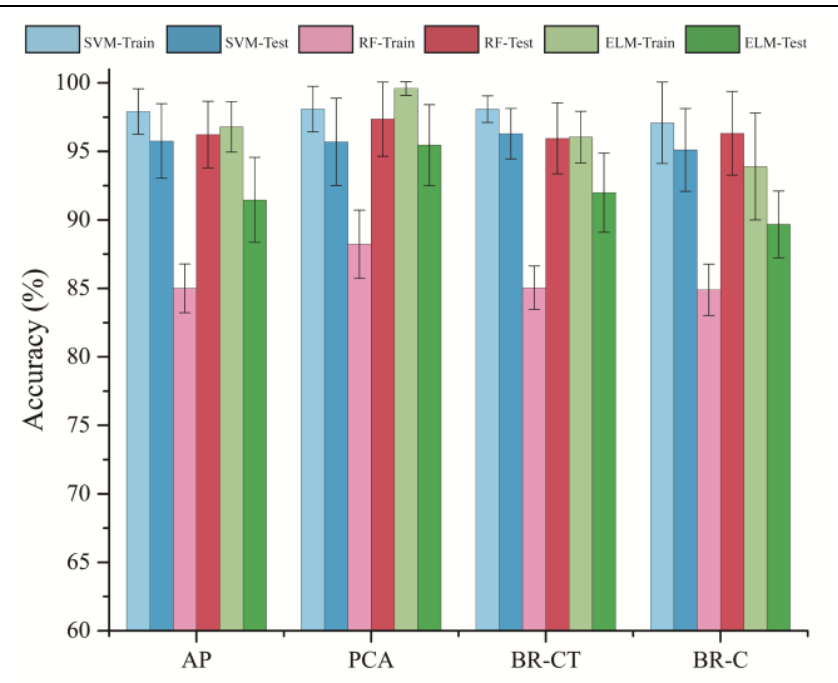

Figure 6. Comparison of average identification accuracies by SVM, RF and ELM models under AP, features extracted by PCA, BR-CT (labeled by 'confirmed' or 'tentative') or BR-C (labeled by 'confirmed' only).

Possibility of Machine-learning-based GC-O. This paper reported a proof-of-concept study aimed at testing the feasibility of ML-based GC-O. 10 ODs were tested due to their relatively larger sample size and higher occurrence frequency in the selected database. A SVM model combined with feature extraction by BR-C showed a high accuracy in the prediction of these ODs from the MPs of odorant molecules. Although 10 descriptors are obviously not enough 
for a practical application, the results of this study demonstrate the possibility that a machine learning approach can be used to obtain sensory information of GC effluents. In fact, the number of prediction models actually needed in a GC-O system may be not as many as expected. A recent study suggested that the dimensionality of odor percepts may be around 20 or less although our nose has 400 olfactory receptors ${ }^{30}$. This may mean 20 or less descriptors are enough for their application in GC-O. It is obvious that more classification models can be expected as long as sufficient data (odorant molecules and corresponding odor descriptors) can be guaranteed. More samples can also ensure the accuracy of the established models. Therefore, the prediction model combined with descriptor indexing may be a good candidate to replace the human panelist in GC-O.

\section{- CONCLUSIONS}

The contribution of this study is to present an approach to predict odor perceptions based on physicochemical descriptors. After processing by the SMOTE and KS methods for balancing dataset and subset partitioning, two feature extraction methods (PCA and BR) were used to extract kernel information from 1006 MPs. Three machine learning approaches (SVM, RF, and ELM) were employed to establish odor descriptor classifier models. The results showed that models calibrated by SVM presented better accuracies than others. Although the accuracy of the BR-C-SVM model is lower than AP-SVM, PCA-SVM, and BR-CT-SVM, when considering the complexities of the models, BR-C-SVM would be the optimal model in this study. Therefore, BR-C-SVM has a good potential in predicting odor perceptions rapidly and precisely. This study demonstrated that MPs associated with machine learning models can be adopted for odor perceptual senses identification. The research is expected to offer a novel approach for developing machine-learning-based GC-O.

\section{ASSOCIATED CONTENT}

Additional information as noted in text. This material is available free of charge via the Internet at http://pubs.acs.org.

\section{AUTHOR INFORMATION}

\section{Corresponding Author}

*Tel: +81 092-802-3629. Fax: +86 092-802-3629. E-mail:

hayashi@ed.kyushu-u.ac.jp

Notes

The authors declare no competing financial interest.

\section{ACKNOWLEDGMENTS}

This research was supported by a grant from the China Scholarship Council (CSC) and JSPS KAKENHI Grant Number $15 \mathrm{H} 01713$.

\section{REFERENCES}

(1) Bartsch, J.; Uhde, E.; Salthammer, T. Anal. Chim. Acta 2016, 904, 98-106.

(2) Brattoli, M.; Cisternino, E.; Dambruoso, P. R.; de Gennaro, G.; Giungato, P.; Mazzone, A.; Palmisani, J.; Tutino, M. Sensors 2013, 13, 16759-16800.

(3) Zellner, B. D.; Dugo, P.; Dugo, G.; Mondello, L. J. Chromatogr. 2008, 1186, 123-143.

(4) Gomez-Miguez, M. J.; Cacho, J. F.; Ferreira, V.; Vicario, I. M.; Heredia, F. J. Food Chem. 2007, 100, 1464-1473.

(5) Casilli, A.; Decorzant, E.; Jaquier, A.; Delort, E. J. Chromatogr. 2014, 1373, 169-178.

(6) Erten, E. S.; Cadwallader, K. R. Food Chem. 2017, 217, 244-253.

(7) Acree, T. E. Anal. Chem. 1997, 69, A170-A175.

(8) Chin, S. T.; Eyres, G. T.; Marriottt, P. J. Anal. Chem. 2012, 84, 9154-9162.

(9) Delahunty, C. M.; Eyres, G.; Dufour, J. P. J. Sep. Sci. 2006, 29, 2107-2125

(10) Guth, H. J. Agric. Food Chem. 1997, 45, 3022-3026.

(11) Pollien, P.; Fay, L. B.; Baumgartner, M.; Chaintreau, A. Anal. Chem. 1999, 71, 5391-5397.

(12) van Ruth, S. M. Biomol. Eng. 2001, 17, 121-128.

(13) Korichi, M.; Gerbaud, V.; Floquet, P.; Meniai, A. H.; Nacef, S.; Joulia, X. In 16th European Symposium on Computer Aided Process Engineering and 9th International Symposium on Process Systems Engineering, Marquardt, W.; Pantelides, C., Eds., 2006, pp 895-900.

(14) Malnic, B.; Hirono, J.; Sato, T.; Buck, L. B. Cell 1999, 96, 713-723. 
(15) Luo, S. X.; Axel, R.; Abbott, L. F. Proc. Natl. Acad. Sci. U.S.A. 2010, 107, 10713-10718.

(16) Johnson, B. A.; Xu, Z.; Ali, S. S.; Leon, M. J. Comp. Neurol. 2009, 514, 658-673.

(17) Haddad, R.; Khan, R.; Takahashi, Y. K.; Mori, K.; Harel, D.; Sobel, N. Nat. Methods 2008, 5, 425-429.

(18) Kumar, R.; Kaur, R.; Auffarth, B.; Bhondekar, A. P. PLoS One 2015, 10, $\mathrm{e} 0141263$.

(19) Keller, A.; Gerkin, R. C.; Guan, Y. F.; Dhurandhar, A.; Turu, G.; Szalai, B.; Mainland, J. D.; Ihara, Y.; Yu, C. W.; Wolfinger, R.; Vens, C.; Schietgat, L.; De Grave, K.; Norel, R.; Stolovitzky, G.; Cecchi, G. A.; Vosshall, L. B.; Meyer, P.; Prediction, D. O. Science 2017, 355, 820-826.

(20) Li, J. Y.; Fong, S. M.; Sung, Y. S.; Cho, K. G.; Wong, R.; Wong, K. K. L. BioData Min. 2016, 9.
(21) Kaneko, H.; Funatsu, K. Chemometrics Intellig. Lab. Syst. 2016, 153, 75-81.

(22) Li, X. H.; Kong, W.; Shi, W. M.; Shen, Q. Chemometrics Intellig. Lab. Syst. 2016, 155, 145-150.

(23) Agjee, N. H.; Ismail, R.; Mutanga, O. J. Appl. Remote Sens. 2016, 10 .

(24) Poona, N. K.; van Niekerk, A.; Nadel, R. L.; Ismail, R. Appl. Spectmsc. 2016, 70, 322-333.

(25) Ren, J. C. Knowl. Based Syst. 2012, 26, 144-153.

(26) Kaeppler, K.; Mueller, F. Chem. Senses 2013, bjs141.

(27) Roweis, S. T.; Saul, L. K. Science 2000, 290, 2323-2326.

(28) Janus, M.; Morawski, A. W. Appl. Catal. B 2007, 75, 118-123.

(29) Cheng, X. P.; Cai, H. M.; Zhang, Y.; Xu, B.; Su, W. F. BMC Bioinformatics 2015, 16.

(30) Meister, M. Elife 2015, 4, e07865. 
Table of contents (TOC) graphic

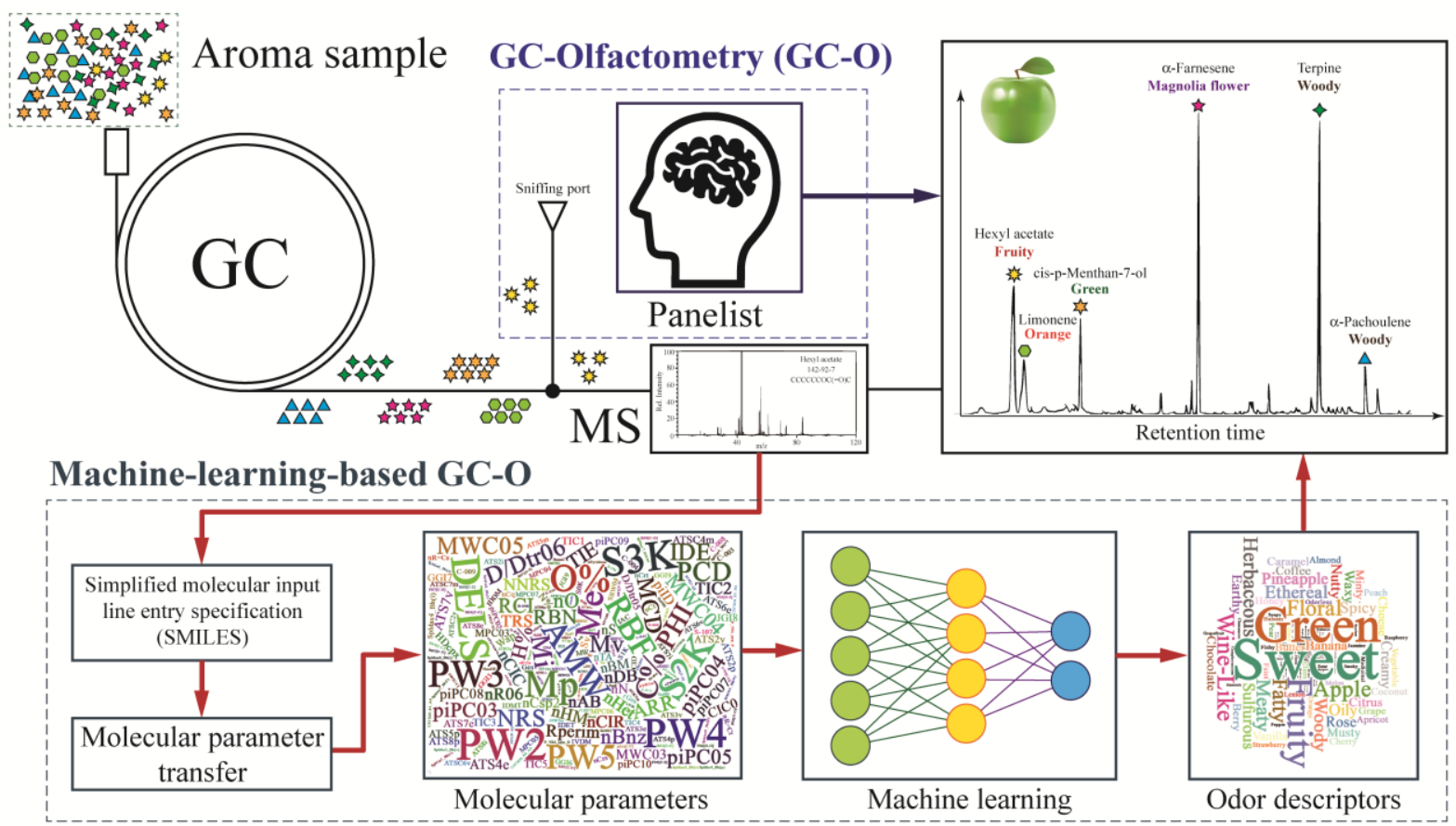

